\title{
Primary oral malignant melanoma metastasis to the brain and breast: A case report and literature review
}

\author{
SAAD ALQAHTANI $^{1}$, AMAL Y. ALHEFDHI $^{2}$, OSAMA ALMALIK $^{2}$, IHAB ANWAR $^{2}$, \\ RANA MAHMOOD $^{3}$, ZEYAD MAHASIN ${ }^{4}$ and TAHER AL-TWEIGERI ${ }^{5}$ \\ ${ }^{1}$ Department of Surgery, College of Medicine, Al Majmaah University, Academic City, Al Majmaah 15341; \\ ${ }^{2}$ Department of General Surgery, King Faisal Specialist Hospital and Research Centre, Alfaisal University; \\ ${ }^{3}$ Department of Radiation Oncology, Oncology Center; ${ }^{4}$ Department of Otolaryngology, Head \\ and Neck Surgery and Communication; ${ }^{5}$ Department of Medical Oncology, Oncology Center, \\ King Faisal Specialist Hospital and Research Centre, Riyadh 11211, Kingdom of Saudi Arabia
}

Received August 2, 2016; Accepted November 30, 2017

DOI: $10.3892 / 01.2017 .6304$

\begin{abstract}
Primary oral malignant melanoma is a rare tumor, which is estimated to comprise $0.2-8.0 \%$ of all melanoma cases. This type of cancer is fairly uncommon, its prognosis is dismal, and it frequently exhibits a biologically aggressive behavior. The common location of primary oral malignant melanoma is the hard palate and maxillary alveolus. In $\sim 85 \%$ of cases, the melanoma will metastasize to the liver, lung, bone and brain early in the course of the disease. The present study reports the case of a 50-year-old premenopausal woman who presented with primary oral malignant spindle cell melanoma (T3bN2aM0) and underwent complete surgical resection followed by an adjuvant course of radiation therapy. After 1 year, the patient presented with sudden onset slurred speech, and upon examination, was found to have left-sided hemiparesis and a hard left breast mass. Workup confirmed breast and brain metastasis. The patient developed lung metastasis 4 weeks later and was referred for palliative care.
\end{abstract}

Correspondence to: Dr Amal Y. Alhefdhi, Department of General Surgery, King Faisal Specialist Hospital and Research Centre, Alfaisal University, P.O. Box 3354 MBC 40, Riyadh 11211, Kingdom of Saudi Arabia

E-mail: alhefdhi@kfshrc.edu.sa

Abbreviations: CT, computed tomography; MRI, magnetic resonance imaging; PET, positron emission tomography; FDG, fluorodeoxyglucose; MBM, melanoma brain metastasis; MAPK, mitogen-activated protein kinase; CTLA-4, cytotoxic T-lymphocyte-associated antigen-4; OS, overall survival; IL-2, interleukin-2

Key words: metastatic melanoma, primary oral malignant melanoma, breast, brain

\section{Introduction}

Melanoma's incidence rates are rising worldwide (1). Melanoma is the third most common type of cancer metastasizing to the brain, subsequent to lung and breast cancer (2). The behavior of cutaneous melanoma is notoriously unpredictable, and the 5-year survival rate deteriorates as the cancer stage progresses $(3,4)$. Morbidity and mortality are mainly associated with metastatic disease, therefore, when metastasis is clinically evident, the prognosis is extremely poor. In total, $90 \%$ of melanoma patients with $\geq 3$ sites of metastasis succumb to disease within 1 year $(3,4)$. Brain metastases are particularly important in the context of malignant melanoma, since $44 \%$ of patients with metastatic melanoma will develop symptomatic brain metastasis (5-7). Intracranial manifestations account for $20-54 \%$ of mortalities in patients with melanoma (5-7). Metastases to the breast from malignant melanoma or from other extra-mammary tumors are rare and represent $\sim 1.3-2.7 \%$ of cases $(8,9)$. Melanoma, lymphoma and lung cancers are the most commonly reported tumors metastasizing to the breast (8-10). In the present study, the case of a premenopausal woman with a history of malignant spindle cell melanoma of the left maxillary alveolus extending to the left hard palate is reported. At 1 year after the initial diagnosis, the patient presented with neurological deficits and a palpable breast mass. The diagnostic and instrumental procedures revealed metastases to the breast, brain and other organs. The patient underwent a right parietal craniotomy and excision of the metastatic melanoma, and planned to start whole-brain radiation and potential immunotherapy (ipilimumab). However, the patient developed multiple metastases and did not receive radiation therapy. The patient was subsequently referred to the palliative care team.

\section{Case report}

The present study reports the case of a 50-year-old premenopausal woman with a negative family history of cancer. The patient had diabetes mellitus type 2 and was receiving oral hypoglycemic medication. In September 2014, the patient 
Table I. Details of the antibodies used in the immunohistochemical staining.

\begin{tabular}{|c|c|c|c|c|c|}
\hline Target protein & Cat. no. & Supplier & Dilution, $\mu \mathrm{g} / \mathrm{ml}$ & Duration, min & Temperature, ${ }^{\circ} \mathrm{C}$ \\
\hline S-100 & $790-2523$ & $\begin{array}{l}\text { Ventana Medical Systems, Inc., } \\
\text { Tucson, AZ, USA }\end{array}$ & $\sim 10.00$ & 24 & 37 \\
\hline Melan-A & $790-2990$ & Ventana Medical Systems, Inc. & $\sim 3.40$ & 24 & 37 \\
\hline MITF & $790-4367$ & Ventana Medical Systems, Inc. & $\sim 6.70$ & 16 & 36 \\
\hline CD34 & $790-2927$ & Ventana Medical Systems, Inc. & $\sim 0.80$ & 24 & 37 \\
\hline p63 & $790-4509$ & Ventana Medical Systems, Inc. & $\sim 0.14$ & 24 & 37 \\
\hline SMA & $760-2833$ & Ventana Medical Systems, Inc. & $\sim 0.02$ & 24 & 37 \\
\hline HMB45 & $790-4366$ & Ventana Medical Systems, Inc. & $\sim 0.50$ & 24 & 37 \\
\hline
\end{tabular}

MITF, microphthalmia-associated transcription factor; CD, cluster of differentiation; SMA, smooth muscle antigen; HMB45, human melanoma black-45.

presented to King Faisal Specialist Hospital (Riyadh, Saudi Arabia) with a swelling of the left maxillary alveolus of 6-month duration, in addition to increased size and intermittent bleeding. Clinically, there was a $3 \times 3-\mathrm{cm}$ irregular, firm and non-tender lesion at the left maxillary alveolus. The lesion extended to the hard palate and buccal mucosa, alongside a large left cervical lymph node. Preoperative computed tomography (CT) scan of the chest, abdomen and pelvis, as well as bone scan and whole-body fluorodeoxyglucose (FDG)-positron emission tomography (PET)/CT scan, revealed a lesion extending to the hard palate and buccal mucosa, which was eroding the maxillary alveolar process. In addition, there was an enlarged necrotic, level-II, left lymph node measuring $3.6 \mathrm{~cm}$, with no evidence of distant or bone metastasis. The patient was diagnosed with a malignant spindle cell melanoma of the left maxillary alveolus extending to the left hard palate. The patient underwent left inferior maxillectomy with obturator and left extended supraomohyoid neck dissection (level I-IV), followed by an adjuvant course of radiation therapy (48 Gy in 20 fractions), which was completed in December 2014. Final histology revealed an undifferentiated malignant melanoma (spindle-cell type). The tumor was $4 \mathrm{~cm}$ in size and $1.5 \mathrm{~cm}$ in thickness. The tumor invaded the submucosa and exhibited prominent lympho-vascular invasion. The bone surrounding margins were free from the tumor. One lymph node was positive for metastatic disease from a total of 62 lymph nodes harvested, with no extra-nodal extension. Molecular characterization by DNA sequencing revealed no mutation in the codon 600 of BRAF (BRAF wild type). No mutation was detected in the C-KIT gene at exons 8, 9, 11 or 17. DNA was extracted manually from a tissue sample. Genetic examinations were performed by polymerase chain reaction using exon 15 specific primers of the BRAF gene. The amplified sequences were then determined using the BigDye terminator sequencing kit and analyzed on an ABI $3730 \mathrm{XL}$ automated sequencer (both supplied by Applied Biosystems; Thermo Fisher Scientific, Inc., Waltham, MA, USA) from the two strands (mutation nomenclature was based on GeneBank accession number NM_004333.4). Immunohistochemical staining was performed using the primary antibodies listed in Table I and the secondary antibody for immunostaining was Ultraview universal DAB detection kit (no. 760-500; Ventana
Medical Systems, Inc., Tucson, AZ, USA) according to the manufacturers protocol. Immunopositivity was demonstrated for S-100 protein, melan A, human melanoma black-45 and microphthalmia-associated transcription factor. However, staining for cluster of differentiation 34, p63 and smooth muscle antigen was negative. Cytogenetic testing for Ewing sarcoma RNA binding protein 1 (22q12) rearrangement was not detected by interphase fluorescence in situ hybridization. Paraffin embedded tissue for Ewing sarcoma tumors are dewaxed with xylene and treated with citric acid in boiling temperature. Treatment was performed using a medical microwave on maximum temperature for $24 \mathrm{~min}$ in $6 \mathrm{~min}$ intervals. Detection of EWSR1 rearrangement was analyzed using Vysis LSI EWSR1 (22q12) Dual Color, Break Apart FISH Rearrangement Probe $(20 \mu \mathrm{l}, 350 \mathrm{ng} / \mu \mathrm{l})$ provided by Abbott Pharmaceutical Co. Ltd., (Probe cat. no. 3N59.20; Lake Bluff, IL, USA) according to manufacturer's protocol. Examination of slide is performed using a fluorescent microscope and 100 cells from each field were analyzed. Postoperatively, the patient recovered well and was subjected to regular follow-up.

In June 2015, the patient presented to King Faisal Specialist Hospital with left-sided hemiparesis and slurred speech of sudden onset, which was associated with ipsilateral jerky movements. On examination, the vital signs were stable, and the patient was fully conscious. No pallor, jaundice, skin rashes or cutaneous lesions were detected. Both pupils (measuring $2 \mathrm{~mm}$ ) were equally reactive. Left-sided weakness was detected with a muscle strength of 3 out of 5, while no cranial or sensory deficit was noticed. A hard mass of $6 \times 6 \mathrm{~cm}$ was observed at the upper outer quadrant of the left breast, which was not attached to the underlying structures. Neither skin or nipple alterations, nor palpable lymph nodes in the axilla, were identified. The right breast and axilla were normal. The rest of the examination was unremarkable.

$\mathrm{CT}$ scan of the brain revealed a heterogonous complex mass with a cystic component, which was located at the left parasagittal posterior parietal region with surrounding edema. The size of the lesion was $2.4 \times 3.2 \mathrm{~cm}$, and the edema extended into the corpus callosum and the contralateral side (Fig. 1). Magnetic resonance imaging (MRI) of the brain revealed a space-occupying lesion with massive peritumoral edema, which was compatible with the findings of the brain CT 


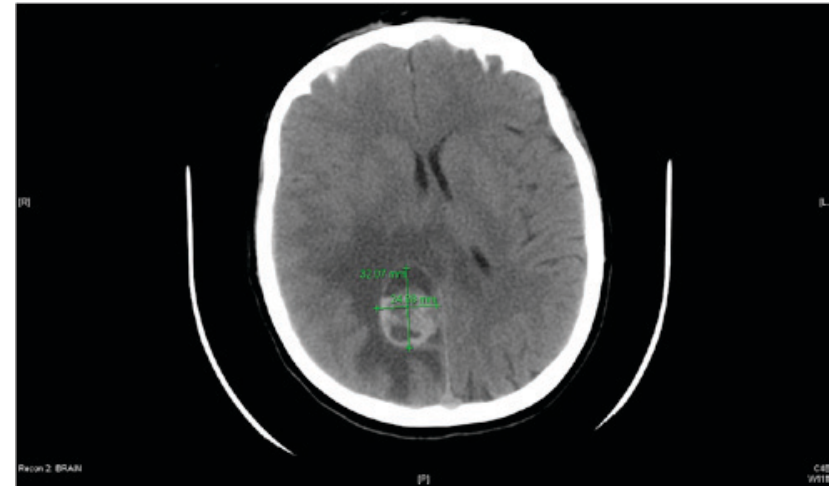

Figure 1. Computed tomography scan of the brain revealed a parietal metastatic lesion. R, right; L, left; P, posterior.

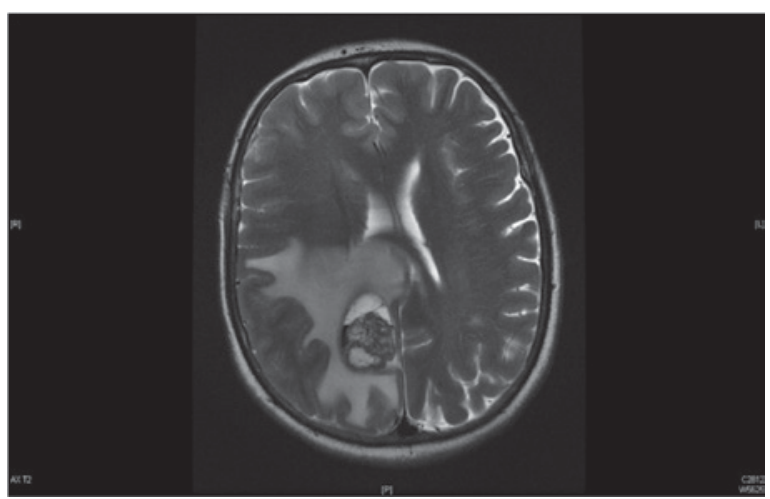

Figure 2. Magnetic resonance imaging of the brain revealed the same parietal metastatic lesion described in Fig. 1, as well as a marked peritumoral edema. $\mathrm{R}$, right; L, left; P, posterior.

scan (Fig. 2). Whole-body FDG-PET/CT scan did not reveal evidence of local recurrence in the left maxilla. However, the lesion in the right cerebral parietal lobe close to the middle line was FDG-avid. There were two newly identified nodules in the left breast compatible with metastasis. In addition, CT scan of the chest, abdomen and pelvis revealed development of a new, enhanced, soft-tissue mass in the left upper outer breast. The mass had a necrotic center, measured 5.6x4.7x7.1 cm and did not present visceral metastasis. Mammogram examination identified a macrolobulated mass in the left breast measuring $5 \times 5 \mathrm{~cm}$ (Fig. 3). Ultrasound examination of the left breast revealed a nonvascular heterogeneous, irregular, macrolobulated mass measuring $5.3 \times 3 \mathrm{~cm}$ (Fig. 4). Several reactive-looking axillary lymph nodes were noted bilaterally. Biopsy of the left breast mass was positive for metastatic malignant melanoma compatible with the primary site.

The patient received anti-seizure medication. Since the brain lesion was symptomatic, relatively large and associated with ample edema, neurosurgical excision followed by immunotherapy and radiation was considered. Radiosurgery could be considered as an alternative option. Thus, the patient underwent right parietal craniotomy and excision of the metastatic melanoma. The final histopathology confirmed the diagnosis of metastatic malignant melanoma. Postoperative CT scan of the brain demonstrated no residual disease. The patient was discharged on October 29, 2015, in good condition, and planned to start whole-brain radiation and potential immunotherapy

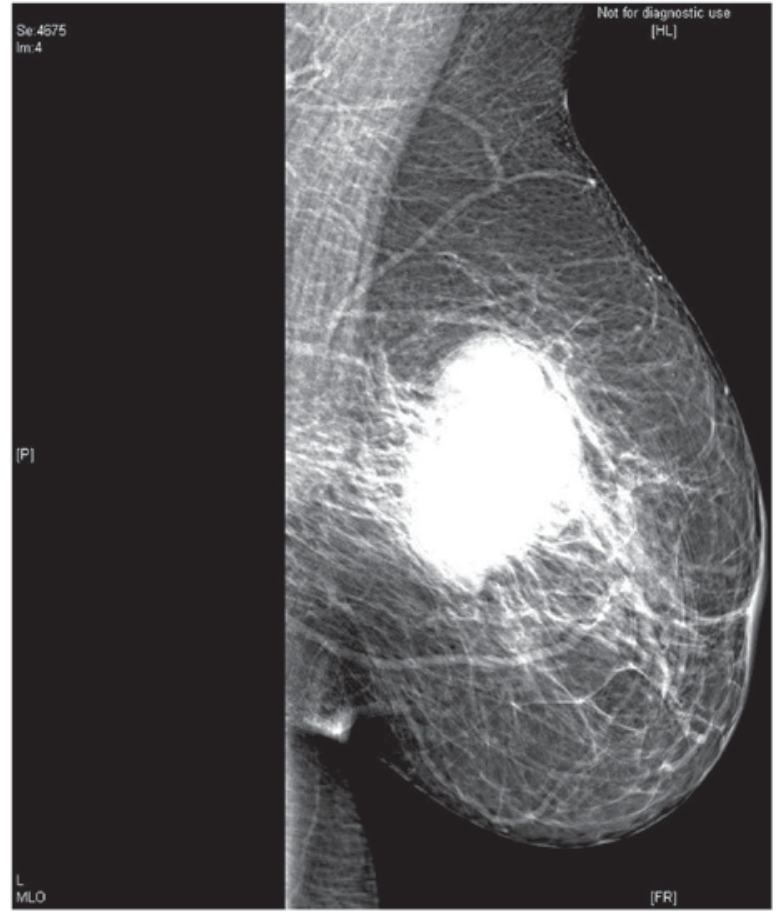

Figure 3. Mammogram of the left breast revealed the presence of an anomalous mass. L, left; P, posterior; MLO, mediolateral-oblique.

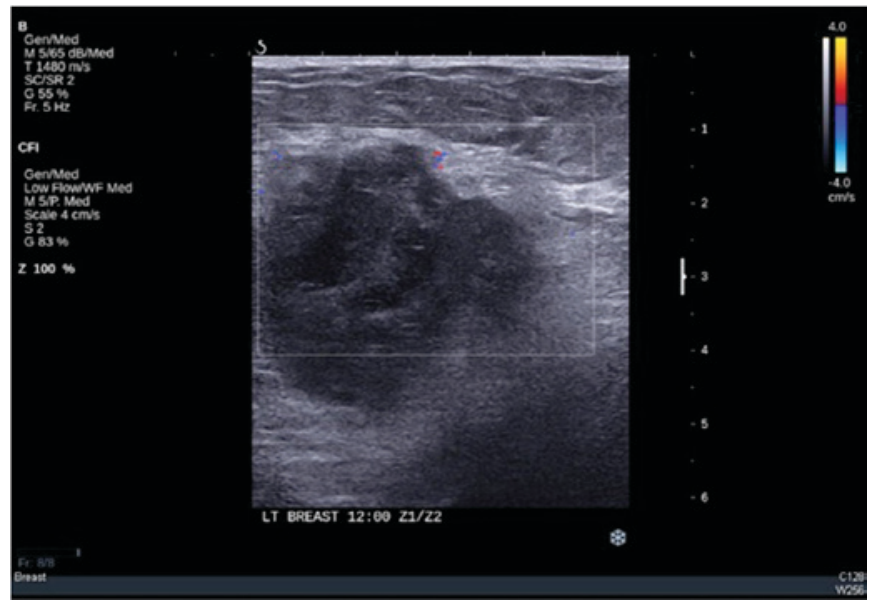

Figure 4. Ultrasound examination of the left breast revealed the presence of an anomalous mass. LT, left; Z1/Z2, zone 1/zone 2.

with the anti-cytotoxic T-lymphocyte-associated antigen 4 (CTLA-4)-blocking agent ipilimumab.

However, 4 weeks later, the patient presented to King Faisal Specialist Hospital through the Emergency Department complaining of headache, right shoulder painful swelling and shortness of breath on mild exertion. On examination, the patient was in respiratory distress, and a tender mass was identified in the right shoulder. The CT scan revealed the left breast mass with skin thickening, presence of new left axillary lymph nodes, enlargement of the mediastinal lymph nodes, development of pericardial effusion and bilateral pleural effusion. Further examination, including MRI of the brain, revealed residual enhanced lesion at the surgical resection bed of the right parietal lobe, and extra-axial enhanced lesion at the right Meckel's cave extending to the right precrural 
cistern, which was consistent with metastasis. Whole-body PET/CT scan demonstrated increased uptake in the brain, left breast, axilla and right shoulder, as well as presence of new mediastinal lymph nodes, bilateral pleural effusion and pericardial effusion. Ultrasound-guided fine needle aspiration of the right shoulder mass was positive for metastatic melanoma. Although radiotherapy and immunotherapy were planned previously, due to the short period of relapse, widespread metastasis and notable deterioration in performance status, on December 2015, the multidisciplinary team at King Faisal Specialist Hospital (including the oncologist, surgeon and palliative care physician) and the patient's family agreed that further active therapy was likely to be futile. Therefore, the patient was transferred to the palliative care team for pain control and end of life care.

\section{Discussion}

Primary oral malignant melanoma is rare, representing $0.2-8.0 \%$ of all melanoma cases (11). The predominant location for this type of cancer is the hard palate and maxillary alveolus (12). It frequently exhibits a markedly aggressive behavior, with rapid growth, high propensity to metastasize and poor prognosis, which is worse than that of cutaneous melanoma (11-13). Independent risk factors that determine the outcome include undifferentiated tumor cell morphology, vascular and neural invasion, tumor necrosis, tumor thickness, and cervical lymph node metastasis (14). In $\sim 85 \%$ of cases, the melanoma will metastasize to the liver, lung, bone or brain early in the course of the disease (15). The reported 5-year survival rate for this cancer ranges from 12 to $16 \%$, with a median survival rate of 18 months after the initial diagnosis (16). Surgery provides the best chance of controlling the disease (17). Chemotherapy and directed targeted therapy are appropriate for patients with unresectable and metastatic disease; however, their role as adjuvant therapies in extensive locoregional disease has not been well defined (11). Moreover, adjuvant radiation therapy is recommended $(18,19)$. The present case exhibited primary oral malignant melanoma with a biological aggressive behavior. In addition, the patient displayed the majority of defined risk factors for this type of cancer and had acceptable definitive local therapy consisting of radical surgery in combination with radiotherapy. However, no adjuvant systemic therapy was administered, in accordance to King Faisal Specialist Hospital and Research Centre institutional guidelines.

Brain metastasis occurs in $10-40 \%$ of all melanoma patients in clinical studies, and in $\leq 90 \%$ in autopsy (20). The brain is the first site of relapse in $10-20 \%$ of these patients (20). Risk factors for developing metastasis include gender (male), presence of a head and neck or oral primary lesion, presence of visceral metastasis, tumor thickness and ulceration of the primary lesion (21). The prognosis of patients with melanoma brain metastasis (MBM) is markedly poor. Prior to the development of anti-CTLA-4/programmed cell death protein 1 antibodies and BRAF/mitogen-activated protein kinase (MAPK)/extracellular signal-regulated kinase inhibitors, the median overall survival (OS) was $\sim 6$ months, with $25 \%$ of patients alive after 1 year (22). The present case had almost all the above risk factors, with the exception of being a female.
MBM were historically managed with surgical resection or whole-brain radiation therapy, depending on the symptoms and the location, number and size of tumors (23). Retrospective data from several reports suggest that melanoma patients with limited brain metastasis treated with surgery and/or stereotactic radiotherapy may achieve better survival than melanoma patients with multiple metastases (23). Temozolomide is the most widely used systemic treatment for MBM; however clinical response was observed only in $10 \%$ of patients $(24,25)$. The introduction of immune checkpoint-blockade antibodies and MAPK inhibitors led to improved survival of metastatic melanoma patients, with a median OS ranging between 10 and 25 months in phase-III studies (26-28). However, patients with brain metastases are underrepresented or excluded from the majority of clinical trials due to historically dismal survival (29); therefore, the impact of these new drugs on the survival of patients with MBM is not well defined. Spagnolo et al (29) recently reported a comprehensive systemic review aimed to analyze the outcomes of patients with MBM treated with immune checkpoint-blockade antibodies (ipilimumab, pembrolizumab and nivolumab) and/or MAPK inhibitors (dabrafenib, vemurafenib and trametinib), regardless of the study design. In total, 22 studies were included with a total of 2,153 patients. The median OS was 7.9 months in phase I-III trials. In safety studies, the OS was 7.0 months for patients treated with immunotherapy vs. 7.9 months for those treated with BRAF inhibitors. In safety studies, the median OS was 4.3 and 7.7 months for patients treated with immunotherapy and BRAF inhibitors, respectively. In that study the authors acknowledged certain limitations in their analysis; despite these limitations, the authors suggested that improved survival may also be achieved in patients with MBM, and supported their inclusion in large clinical trials (29). The current case was unable to receive ipilimumab due to early onset of widespread metastasis and marked rapid decline in the patient's performance status, with subsequent early referral to a palliative care program.

The frequency of metastatic tumors to the breast from extra-mammary malignancy based on histological diagnosis varies between 0.2 and $1.3 \%$ in clinical studies and between 0.2 and $6 \%$ in autopsy studies (30). The demographics and clinical features are similar to those of primary breast cancers, but the prognosis and management options are often different. In $30 \%$ of patients, metastasis to the breast is the first sign of malignancy (30-32). Patients typically present with a rapidly growing, painless, firm mass. In addition, diffuse skin involvement is rare, and axillary lymph nodes are uncommon, similarly to the current patient's presentation $(30,31,33,34)$.

Clinical examination and imaging features of breast metastases from melanoma usually do not allow a differential diagnosis from breast primary tumors. The most common mammographic appearance is a rounded mass with well-defined or slightly irregular margins, while microcalcification is rare $(30,34,35)$. Ultrasound scans typically reveal a hypoechoic mass, which is usually heterogeneous and poorly defined (35). In a review of patients presenting with metastases to the breast from extra-mammary tumors, Toombs and Kalisher noticed that $50 \%$ of metastases were located in the upper outer quadrant of the breast, similar to the current case; however, the position of a lump in the breast does not aid to 
distinguish primary from secondary malignancies (31). It has been reported that patients with breast metastases from melanoma are often young and premenopausal (36), which supports the hypothesis of hormonally driven progression of melanoma. Although the influence of estrogen in the development and progression of melanoma has been debated, the number of epidemiological studies implicating estrogen in the etiology of melanoma has increased (37).

Treatment for metastatic breast cancer differs greatly from that for primary breast cancer and, due to the poor prognosis of metastatic disease, the treatment should be individualized. In patients with isolated metastatic disease limited to the breast or with minimal disease burden, wide local excision may be considered. Large bulky tumors may be palliated by mastectomy, although this procedure should be avoided where possible. In the past, the treatment options for metastatic melanoma were limited. Chemotherapeutic agents and/or biological response modifiers such as interleukin-2 (IL-2) are ineffective. The response rate to a high dose of IL-2 has been reported to be $\sim 7 \%$, and long-term survival is observed only in $8 \%$ of stage-IV patients, irrespective of the site of metastases. However, the use of high-dose IL-2 was restricted due to critical side effects on multiple organ systems (38). Recently, the introduction of molecularly targeted therapy in parallel with the development of checkpoint inhibitors has rapidly improved the outcomes of metastatic melanoma patients (38).

In conclusion, the case reported in the present study highlights the importance of early diagnosis and the poor outcomes for patients with primary oral malignant melanoma, which is a rare disease. Biologically, this type of cancer has an aggressive behavior and poor survival. However, in the majority of cases, the diagnosis is delayed until symptoms such as swelling, ulceration and bleeding occur. Thus, early detection of oral melanoma is critical. Due to the poor survival of patients with this disease, clinical trials of novel systemic therapy using targeted or immune modulators agents should consider enrolling primary oral malignant melanoma patients, regardless of the apparent extent of the disease.

\section{References}

1. Little EG and Eide M: Update on the recurrence state of melanoma incidence. Dematol Clin 30: 355-361, 2012.

2. Patchell RA: The management of brain metastasis. Cancer Treat Rev 29: 533-540, 2003.

3. Balch CM, Houghton AN, Sober AJ and Soong SJ: Cutaneous Melanoma. 4th edition. Quality Medical Publishing, St. Louis, MO, USA, 2003.

4. Balch CM, Gershenwald JE, Soong SJ, Thompson JF, Atkins MB, Byrd DR, Buzaid AC, Cochran AJ, Coit DG, Ding S, et al: Final version of 2009 AJCC melanoma staging and classification. J Clin Oncol 27: 6199-6206, 2009.

5. Budman DR, Camacho E and Wittes RE: The current causes of death in patients with malignant melanoma. Eur J Cancer 14: 327-330, 1978.

6. Davies MA, Liu P, McIntyre S, Kim KB, Papadopoulos N, Hwu WJ, Hwu P and Bedikian A: Prognostic factors for survival in melanoma patients with brain metastases. Cancer 117: 1687-1696, 2011.

7. Patel JK, Didolkar MS, Pickern JW and Moore RH: Metastatic pattern of malignant melanoma: A study of 216 autopsy cases. Am J Surg 135: 807-810, 1978.

8. Ravdel L, Robinson WA, Lewis K and Gonzalez R: Metastatic melanoma in the breast: A report of 27 cases. J Surg Oncol 94 101-104, 2006.

9. Al Samaraee A, Khout H, Barakat T and Fasih T: Breast Metastasis from a melanoma. Ochsner J 12: 149-151, 2012.
10. Thomson JF, Scolyer RA and Kefford RF: Cutaneous melanoma. Lancet 365: 687-701, 2005.

11. Rapidis AD, Apostolidis C, Vilos G and Valsamis S: Primary malignant melanoma of the oral mucosa. J Oral Maxillofac Surg 61: 1132-1139, 2003.

12. Shen ZY, Liu W, Bao ZX, Zhou ZT and Wang LZ: Oral melanotic macule and primary oral malignant melanoma: Epidemiology, location involved, and clinical implications. Oral Surg Oral Med Oral pathol Oral Radiol Endod 112: e21-e25, 2011.

13. Patel SG, Prasad ML, Escrig M, Singh B, Shaha AR, Kraus DH, Boyle JO, Huvos AG, Busam K and Shah JP: Primary mucosal malignant melanoma of the head and neck. Head Neck 24: 247-257, 2002.

14. Keller DS, Thomay AA, Gaughan L, Olszanski A, Wu H, Berger AC and Farma JM: Outcomes in patients with mucosal melanomas. J Surg Oncol 108: 516-520, 2013.

15. Tlholoe MM, Khammissa RA, Bouckaert M, Altini M, Lemmer J and Feller L: Oral mucosal melanoma: Some pathobiological considerations and illustrative report of a case. Head Neck Pathol 9: 127-134, 2015.

16. Kumar K, Santhosh BS and Priya NK: Primary oral malignant melanoma-a case report. Nig Dent J 19: 44-47, 2011.

17. Jayaraj SM, Hern JD, Mochloulis G and Porter GC: Malignant melanoma arising in the frontal sinuses. J Laryngol Otol 111: 376-378, 1997.

18. Thompson AC, Morgan DA and Bradely PJ: Malignant melanoma of the nasal cavity and paranasal sinuses. Clin Otolaryngol Allied Sci 18: 34-36, 1993.

19. Gilligan D and Selvin NJ: Radical radiotherapy for 28 cases of mucosal melanoma in the nasal cavity and sinuses. Br J Radiol 64: 1147-1150, 1991.

20. Chiarion-Sileni V, Murr R, Pigozzo J, Sarti S, Tomassi O and Romanini A: Brain metastases from malignant melanoma. Forum (Genova) 13: 170-182, 2003.

21. Cohn-Cedermark G, Måsson-Brahme E, Rutqvist LE, Larsson O, Johansson $\mathrm{H}$ and Ringborg U: Central nervous system metastases of cutaneous malignant melanoma-a population based study. Acta Oncol 37: 463-470, 1998.

22. Korn EL,Liu PY,Lee SJ, Chapman JA, Niedzwiecki D, Suman VJ, Moon J, Sondak VK, Atkins MB, Eisenhauer EA, et al: Meta-analysis of phase II cooperative group trials in metastatic stage IV melanoma to determine progression-free survival and overall survival benchmarks for future phase II trials. J Clin Oncol 26: 527-534, 2008.

23. Vecchio S, Spagnolo F, Merlo DF, Signori A, Acquati M, Pronzato P and Queirolo P: The treatment of melanoma brain metastases before the advent of targeted therapies: Associations between therapeutic choice, clinical symptoms and outcome with survival. Melanoma Res 24: 61-67, 2014.

24. Agarwala SS, Kirkwood JM, Gore M, Dreno B, Thatcher N, Czarnetski B, Atkins M, Buzaid A, Skarlos D and Rankin EM: Temozolomide for treatment of brain metastases associated with metastatic melanoma: A phase II study. J Clin Oncol 22: 2101-2107, 2004.

25. Margolin K, Atkins M, Thomson J, Ernstoff S, Weber J, Flaherty L, Clark I, Weiss G, Sosman J, II Smith W, et al: Temozolomide and whole brain radiation in melanoma metastatic to the brain: A phase II trial of the cytokine working group. J Cancer Res Clin Oncol 128: 214-218, 2002.

26. Fodi FS, O'Day SJ, McDermott DF, Weber RW, Sosman JA, Haanen JB, Gonzalez R, Robert C, Schadendorf D, Hassel JC, et al: Improved survival with ipilimumab in patients with metastatic melanoma. N Engl J Med 363: 711-723, 2010.

27. McArthur GA, Chapman PB, Robert C, Larkin J, Haanen JB, Dummer R, Ribas A, Hogg D, Hamid O, Ascierto PA, et al: Safety and efficacy of vemurafenib in BRAF(V600E) and BRAF(V600K) mutation-positive melanoma (BRIM-3): Extended follow-up of a phase 3, randomised, open-label study. Lancet Oncol 15: 323-332, 2014.

28. Long GV, Stroyakovskiy D, Gogas H, Levchenko E, de Braud F, Larkin J, Garbe C, Jouary T, Hauschild A, Grob JJ, et al: Dabrafenib and trametinib versus dabrafenib and placebo for Val600 BRAF-mutant melanoma: A multicenter, double blind, phase 3 randomised controlled trial. Lancet 386: 444-451, 2015.

29. Spagnolo F, Picasso V, Lambertini M, Ottaviano V, Dozin B and Queirolo P: Survival of patients with metastatic melanoma and brain metastases in era of MAP-kinase inhibitors and immunologic checkpoint blockade antibodies: A systemic review. Cancer Treat Rev 45: 38-45, 2016. 
30. Alvarado Cabrero I, Carrera Alvarez M, Pérez Montiel D and Tavassoil FA: Metastases to the breast. Eur J Surg Oncol 29 : 854-855, 2003.

31. Toombs BD and Kalisher L: Metastatic disease to the breast: Clinical, pathologic, and radiographic features. AM J Roentgenol 129: 673-676, 1977

32. Georgiannos SN, Chin J, Goode AW and Sheaff M: Secondary neoplasms of the breast: A survey of the 20th century. Cancer 92 2259-2266, 2001.

33. Amichetti M, Perani B and Boi S: Metastases to the breast from extra-mammary malignancies. Oncology 47: 257-260, 1990.

34. Vergier B, Trojani M, de Mascarel I, Coindre JM and Le Treut A: Metastases to the breast: Differential diagnosis from primary breast carcinoma. J Surg Oncol 48: 112-116, 1991.
35. Lee SH, Park JM, Kook SH, Han BK and Moon WK: Metastatic tumors to the breast: Mammographic and ultrasonographic findings. J Ultrasound Med 19: 257-262, 2000.

36. Arora R and Robinson WA: Breast metastases from malignant melanoma. J Surg Oncol 50: 27-29, 1992.

37. Miller JG and Mac Neil S: Gender and cutaneous melanoma. $\mathrm{Br}$ J Dermatol 136: 657-665, 1997.

38. Davey RJ, van der Westhuizen A and Bowden NA: Metastatic melanoma treatment: Combining old and new therapies. Crit Rev Oncol Hematol 98: 242-253, 2016. 